\title{
Segmentation of Longitudinal Brain MR Images Using Bias Correction Embedded Fuzzy C-Means with Non-locally Spatio-temporal Regularization
}

\author{
Chaolu Feng,a,b,*, Dazhe Zhao, ${ }^{\mathrm{a}, \mathrm{b}}$, Min Huang $\mathrm{g}^{\mathrm{a}, \mathrm{c}}$ \\ ${ }^{a}$ School of Computer Science and Engineering, Northeastern University, Shenyang, Liaoning, 110819, China \\ ${ }^{b}$ Key Laboratory of Medical Image Computing of Ministry of Education, Northeastern University, Shenyang, Liaoning, \\ 110819, China \\ ${ }^{c}$ State Key Laboratory of Synthetical Automation for Process Industries, Northeastern University, Shenyang, Liaoning, \\ 110819, China
}

\begin{abstract}
We propose an automated method for segmentation of brain tissues in longitudinal MR images. In the proposed method, images acquired at each time point are first separately segmented into white matter, gray matter, and cerebrospinal fluid by bias correction embedded fuzzy C-means. Intensities differences are then defined as similarities of each voxel to the cluster centroids. After being normalized in inter-class, the similarities are incorporated into a non-local means de-noising formula to regularize the segmentation in both spatial and temporal dimensions. Non-locally regularization results are used to compute final membership functions for the segmentation. To improve time performance, we accelerate the modified denoising algorithm using CUDA and obtain a $200 \times$ performance improvement. Quantitative comparison with the state-of-the-art methods on BrainWeb dataset demonstrate advantages of the proposed method in terms of segmentation accuracy and the ability to consistently segment brain tissues in an arbitrary number of longitudinal brain MR image series.
\end{abstract}

Keywords: longitudinal segmentation, brain atrophy, non-local means de-noising, intensity inhomogeneity, CUDA

\section{1. Introduction}

Brain atrophy, observed in a variety of neurodegenerative disorders, such as multiple sclerosis (MS), Alzheimer's disease (AD), Huntington's disease, and Parkinson's disease, generally manifests itself as vol4 ume changes in brain tissues $[1,2]$. Due to its non-invasive nature and excellent soft tissue contrast, magnetic 5 resonance imaging (MRI) has been widely used by various image processing technologies, such as segmentation, to reveal changes of human brain in basic and clinical neuroscience studies [3, 4]. Thus, segmentation of magnetic resonance (MR) images of human brain into anatomically meaningful tissues such as white matter

\footnotetext{
* Corresponding author

Email address: fengchaolu@cse.neu.edu.cn (Chaolu Feng)
} 
(WM), gray matter (GM), and cerebrospinal fluid (CSF) is crucial for measurement of subtle but complex changes of human brain quantitatively $[5,6]$.

Since it is labour-intensive, time-consuming, and sensitive to inter- and intra-observer variability, manual segmentation suffers from lacks of reproducibility and ability of maintaining consistency for entire dataset of a study $[7,6]$. Therefore, many efforts have been devoted to developing automated methods in the literature for analysis of brain atrophy in $\operatorname{MR}$ images $[8,9,10,11,12,13,14,15,16,17]$. But most of the methods are proposed merely in view of cross-sectional (3D) images of human brain $[8,9,10,11,12,13]$. It is obvious that 3D segmentations are not able to provide adequate longitudinal stability because images at each single time point are segmented separately $[18,19,20]$. In contrast, segmentation of a series of $3 \mathrm{D}$ image sequences of the same subject simultaneously has been shown to be able to increase the accuracy of brain atrophy measurement $[21,3]$. That is to say that segmentation of longitudinal brain MR images (images at multiple time points) consistently is crucial to the measurement of subtle volume changes of brain tissues [22, 23]. In [24], Smith et al. presented a fully automated method to analyse changes of human brain longitudinally. They first automatically distinguish brain tissues from non-brain tissues and then registered the brain MR images while estimated skull images were used to constrain scaling and skew. They finally estimated brain surface motion by tracking surface points of the brain in subvoxel accuracy $[25,26]$. This method has already been incorporated into a soft package named SIENA (http: //fsl.fmrib.ox.ac.uk/fsl/fslwiki/SIENA) for analysis of brain change in both single-time-point (crosssectionally) and two-time-point (longitudinally). In [12], Zhang et al. proposed a method to segment brain MR images by using a hidden Markov Random Field (MRF) model and the Expectation-Maximization (EM) algorithm. Their method has been generalized and integrated into FMRIB Software Library (FSL) as a tool named FMRIB's Automated Segmentation Tool (FAST, http://fsl.fmrib.ox.ac.uk/fsl/fslwiki/FAST) with the ability of correcting the spatial intensity inhomogeneities and simultaneously segmenting the brain image into WM, GM, and CSF. As a brain imaging software package developed by the Martinos Center, Freesurfer (FSF, http://freesurfer.net/) includes volumetric segmentation and longitudinal processing functions for brain image analysis [27]. The segmentation method in Freesurfer is based on voxel intensities and Talairach anatomical atlases where images are automatically processed for longitudinal analysis within the longitudinal stream to extract reliable volume estimations [28]. Xue et al. proposed a temporally consistent and spatially adaptive longitudinal MR brain image segmentation method named CLASSIC in [29].Their method incorporates image-adaptive clustering, spatio-temporal smoothness constraints, and image warping to jointly segment a series of brain MR images of the same subject aiming at obtaining accurate measurements of rates of change of regional and global brain volumes from serial MR images and estimating the morphological changes of the brain, such as atrophy [29]. Although a few longitudinal segmentation methods have been proposed in the literature, segmentation of MR brain images longitudinally is still an open problem and is challenging due to the large size of brain MR images especially at multiple time 
points, the limitation of available a priori knowledge, and the presence of noise and intensity inhomogeneities (well known as bias field) [30]. Fig. 1 shows an example of intensity overlaps between different brain tissues caused by noise and intensity inhomogeneity.
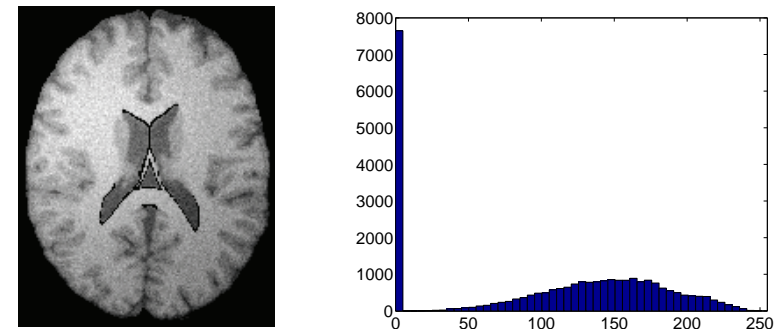

Figure 1: Appearance and intensity histograms of a brain image corrupted by both noise and intensity inhomogeneity.

As one of the well known clustering methods, fuzzy c-means (FCM) has been widely studied in the literature for its application to image segmentation [31]. But traditional FCM and hard c-means are both sensitive to noise and lacks the ability to deal with intensity inhomogeneity[32]. To overcome this drawback, Ahmed et al. proposed a bias corrected fuzzy c-means (BCFCM) method by taking consideration of the correlation among neighbouring pixels [33]. Chen and Zhang modified BCFCM to improve its time performance and robustness to noise and outliers [34]. However, final clustering results of methods proposed by Ahmed et al. and Chen and Zhang are both heavily affected by a parameter used to adjust influence of the neighbouring pixels. Therefore, Yang and Tsai proposed a generalized type of BCFCM in [35] where the parameter is estimated automatically under a learning scheme. However, all the above mentioned improvements of BCFCM are without pay any attention to correct the bias field from the image. In this paper, we propose a fully automated method for segmentation of brain tissues longitudinally. Besides being able to deal with the intensity inhomogeneities inherently, the proposed method is effective in segmenting brain tissues in arbitrary number of MR image series. In the method, a bias correction embedded FCM (BCEFCM) is first proposed to segment 3D MR image sequences of each time point separately and estimate the bias field simultaneously. A non-locally spatio-temporal regularization is then introduced to ensure segmentation consistency of the proposed method. The regularization is defined on similarities of intensities between voxels and cluster centroids. Specifically, after being normalized in inter-class, the similarities are incorporated into a non-local means de-noising formula by summing them together and introducing a time component integral to regularize longitudinal images non-locally in both spatial and temporal dimensions. It is obvious that the proposed method is also able to overcome negative influences of noise as well because of the existing of non-local means de-noising. Membership functions are finally updated with non-local means de-noising results, and are further used to decide which class the voxels should be classified into.

The rest of this paper is organized as follows. The framework and formulation of the proposed method are described in section 2. Experimental results, quantitative evaluation, and discussions are given in section 
3. This paper is finally concluded in section 5 .

\section{Method}

In this paper, we suppose that the MR images of different time points have already been rigidly registered in the same coordinate system and non-brain tissues have already been removed from the images. For the images used in this paper, maximization of mutual information and a fast robust automated brain extraction tool, BET, are used to register each image case and strip non-brain tissues from the images, respectively $[36,37]$. Before performing segmentation, longitudinal MR image series denoted by $\mathbf{I}=\left(I_{1}, I_{2}, \ldots, I_{T}\right)$, which include $T$ image sequences, are first rigidly registered to the first image sequence in the same image domain $\Omega$ with six degree of freedom. Given a brain MR image $I_{t}$ acquired at time point $t \in[1, T]$, we view it as a function $I_{t}: \Omega \rightarrow R$ defined on the continuous image domain $\Omega \subset R^{2}$. It has been well known that the observed image can be described by a product of the true image intensity $J_{t}$ and the unknown bias field $b_{t}$, namely,

$$
I_{t}(\mathbf{x})=b_{t}(\mathbf{x}) J_{t}(\mathbf{x})+n_{t}(\mathbf{x})
$$

where $\mathbf{x} \in \Omega$ and $n_{t}$ is zero-mean additive noise. The true image $J_{t}$ characterizes an intrinsic physical property of brain tissues, which ideally takes a specific intensity $c_{t, i}$ for the $i$-th type of tissues and is therefore assumed to be piecewise constant. That is to say, the true image $J_{t}$ approximately takes $N$ distinct constant values $c_{t, 1}, c_{t, 2}, \ldots$, and $c_{t, N}$ in disjoint regions $\Omega_{t, 1}, \Omega_{t, 2}, \ldots$, and $\Omega_{t, N}$, respectively. The bias field $b$ is assumed to be slowly and smoothly varying as well known in the literature. The problem of image segmentation is therefore considered as finding the specific intensities $c_{1}, c_{2}, \ldots$, and $c_{N}$ and estimating the bias field $b$ simultaneously.

\subsection{Bias Correction Embedded Fuzzy C-Means (BCEFCM)}

As mentioned above, the true image $J_{t}$ is assumed to be piecewise constant and therefore takes a specific intensity $c_{t, i}$ for the $i$-th type of brain tissues in the region $\Omega_{t, i}$. Thus, if $\mathbf{x}$ is most probably classified as the $i$-th type of tissues, then the intensity of the true image $J_{t}$ at pixel $\mathbf{x}$ is close to the constant $c_{t, i}$, i.e.

$$
J_{t}(\mathbf{x}) \approx c_{t, i} \quad \text { for } \quad \mathbf{x} \in \Omega_{\mathrm{t}, \mathrm{i}}
$$

Then, in view of the image model in Eq. (1), we have

$$
I_{t, i}(\mathbf{x}) \approx b_{t}(\mathbf{x}) c_{t, i}+n_{t}(\mathbf{x}) \quad \text { for } \quad \mathbf{x} \in \Omega_{\mathrm{t}, \mathrm{i}}
$$

Taking into account that $n_{t}$ is zero-mean additive noise, the intensities in the set

$$
I_{t, i}=\left\{I(\mathbf{x}): \mathbf{x} \in \Omega_{t, i}\right\}
$$


form a cluster with the cluster centroid $m_{t, i} \approx b_{t}(\mathbf{x}) c_{t, i}$. This clustering property indicates that the intensities in the image domain $\Omega$ can be classified into $N$ clusters with centroids $m_{t, 1} \approx b_{t}(\mathbf{x}) c_{t, 1}, m_{t, 2} \approx b_{t}(\mathbf{x}) c_{t, 2}, \ldots$, and $m_{t, N} \approx b_{t}(\mathbf{x}) c_{t, N}$, respectively. To classify these intensities, we define

$$
F_{t}\left(b_{t}, \mathbf{u}_{t}, \mathbf{c}_{t}\right)=\int_{\Omega} \sum_{i=1}^{N}\left\|I_{t}(\mathbf{x})-b_{t}(\mathbf{x}) c_{t, i}\right\|^{2} u_{t, i}(\mathbf{x}) d \mathbf{x}
$$

where $u_{t, i}$ is the membership function of the region $\Omega_{t, i}, \mathbf{c}_{t}=\left(c_{t, 1}, c_{t, 2}, \ldots, c_{t, N}\right), \mathbf{u}_{t}=\left(u_{t, 1}, u_{t, 2}, \ldots, u_{t, N}\right)$, and $\|*\|$ is one norm expression of the similarity (Euclidean distance in general) between measured intensity $I_{t}(\mathbf{x})$ and the cluster centroid $b_{t}(\mathbf{x}) c_{t, i}$.

In the ideal case, each voxel either belongs to one tissue or not, such that $u_{t, i}(\mathbf{x})=1$ for $\mathbf{x} \in \Omega_{t, i}$ and $u_{t, i}(\mathbf{x})=0$ for $\mathbf{x} \notin \Omega_{t, i}$, which will introduce a hard segmentation. In reality, one voxel may contain more than one type of tissues due to the partial volume effect, especially at the interface between neighboring tissues. In this case, the $N$ tissues can be represented by fuzzy membership functions $u_{t, i}(\mathbf{x})$ that take values between 0 and 1 and satisfy $\sum_{i=1}^{N} u_{t, i}(\mathbf{x})=1$. The value of the fuzzy membership function $u_{t, i}(\mathbf{x})$ can be interpreted as the percentage of the $i$-th tissue within the voxel $\mathbf{x}$. To achieve fuzzy segmentation, we rewrite the energy function $F_{t}$ defined above by introducing a fuzzifier $q \geq 1$ as follows

$$
F_{t}\left(b_{t}, \mathbf{u}_{t}, \mathbf{c}_{t}\right)=\int_{\Omega} \sum_{i=1}^{N}\left\|I_{t}(\mathbf{x})-b_{t}(\mathbf{x}) c_{t, i}\right\|^{2} u_{t, i}^{q}(\mathbf{x}) d \mathbf{x}
$$

To generalize the above defined formulation and provide an option for users to improve the segmentation result, we introduce positive weighting coefficients $\lambda_{1}, \lambda_{2}, \ldots, \lambda_{N}$ for the $N$ clusters and modified the above energy as follows

$$
F_{t}\left(b_{t}, \mathbf{u}_{t}, \mathbf{c}_{t}\right)=\int_{\Omega} \sum_{i=1}^{N} \lambda_{i}\left\|I_{t}(\mathbf{x})-b_{t}(\mathbf{x}) c_{t, i}\right\|^{2} u_{t, i}^{q}(\mathbf{x}) d \mathbf{x}
$$

This objective function is minimized when high membership values are assigned to voxels, intensities of which are close to the centroid, and low membership values are assigned when the voxels are far from the centroids.

Remark 1: It is worth pointing that the objective function defined above is a special case of objective functions in BCFCM and FCM. That is to say the proposed BCEFCM is a generalization of the well-known FCM. Note that for all other parameters fixed, the smaller the parameter $\lambda_{i}$ is, the greater the $i$-cluster is, and vice versa. We will discuss the impact of parameter $\lambda_{i}$ on the size of the $i$-th cluster in section 4

\subsection{Energy Minimization}

Segmentation of the brain MR image $I_{t}$ can be performed by minimizing the energy $F_{t}\left(b_{t}, \mathbf{u}_{t}, \mathbf{c}_{t}\right)$ defined in Eq.(7) subject to the constraints $0 \leq u_{t, i}(\mathbf{x}) \leq 1$ and $\sum_{i=1}^{N} u_{t, i}(\mathbf{x})=1$ for all $\mathbf{x} \in \Omega$. The minimization can be achieved by alternately minimizing energy function $F_{t}\left(b_{t}, \mathbf{u}_{t}, \mathbf{c}_{t}\right)$ with respect to each of its variables as follows. 
For fixed $b_{t}(\mathbf{x})$ and $u_{t, i}(\mathbf{x})$ where $i=1,2, \ldots, N$, we minimize $F_{t}\left(b_{t}, \mathbf{u}_{t}, \mathbf{c}_{t}\right)$ with respect to $c_{t, i}$ by resolving $\frac{\partial F_{t}\left(b_{t}, \mathbf{u}_{t}, \mathbf{c}_{t}\right)}{\partial c_{t, i}}=0$. It is obvious that $F_{t}\left(b_{t}, \mathbf{u}_{t}, \mathbf{c}_{t}\right)$ is minimized at $c_{t, i}=\hat{c}_{t, i}$, given by

$$
\hat{c}_{t, i}=\frac{\int_{\Omega} b_{t}(\mathbf{x}) I_{t}(\mathbf{x}) u_{t, i}^{q}(\mathbf{x}) d \mathbf{x}}{\int_{\Omega} b_{t}^{2}(\mathbf{x}) u_{t, i}^{q}(\mathbf{x}) d \mathbf{x}}
$$

For fixed $u_{t, i}(\mathbf{x})$ and $c_{t, i}$ where $i=1,2, \ldots, N$, we minimize $F_{t}\left(b_{t}, \mathbf{u}_{t}, \mathbf{c}_{t}\right)$ with respect to $b_{t}(\mathbf{x})$ by resolving $\frac{\partial F_{t}\left(b_{t}, \mathbf{u}_{t}, \mathbf{c}_{t}\right)}{\partial b_{t}(\mathbf{x})}=0$. It can be shown that $F_{t}\left(b_{t}, \mathbf{u}_{t}, \mathbf{c}_{t}\right)$ is minimized at $b_{t}(\mathbf{x})=\hat{b}_{t}(\mathbf{x})$, given by

$$
\hat{b}_{t}(\mathbf{x})=\frac{I_{t}(\mathbf{x}) \sum_{i=1}^{N} \lambda_{i} c_{t, i} u_{t, i}^{q}(\mathbf{x})}{\sum_{i=1}^{N} \lambda_{i} c_{t, i}^{2} u_{t, i}^{q}(\mathbf{x})}
$$

For the case $q>1$, minimization of $F_{t}\left(b_{t}, \mathbf{u}_{t}, \mathbf{c}_{t}\right)$ with respect to $u_{t, i}(\mathbf{x})$ can be implemented by resolving the following Lagrangian equation:

$$
\sum_{i=1}^{N} \lambda_{i} \int_{\Omega}\left\|I_{t}(\mathbf{x})-b_{t}(\mathbf{x}) c_{t, i}\right\|^{2} u_{t, i}^{q}(\mathbf{x}) d \mathbf{x}-\lambda\left(\sum_{i=1}^{N} u_{t, i}(\mathbf{x})-1\right)=0
$$

where $\lambda$ is the Lagrangian multiplier and $\sum_{i=1}^{N} u_{t, i}(\mathbf{x})=1$ is the extremum condition. For fixed $b_{t}(\mathbf{x})$ and $c_{t, i}$ where $i=1,2, \ldots, N$, we take partial derivative of the above equation with respect $u_{t, i}(\mathbf{x})$, set the result to 0 , and resolve the equations with the constraint that $\sum_{i=1}^{N} u_{t, i}(\mathbf{x})=1$. Then, it can be shown that $F_{t}\left(b_{t}, \mathbf{u}_{t}, \mathbf{c}_{t}\right)$ is minimized at $u_{t, i}(\mathbf{x})=\hat{u}_{t, i}(\mathbf{x})$, given by

$$
\hat{u}_{t, i}(\mathbf{x})=\frac{\left(\lambda_{i}\left\|I_{t}(\mathbf{x})-b_{t}(\mathbf{x}) c_{i}\right\|^{2}\right)^{\frac{1}{1-q}}}{\sum_{j=1}^{N}\left(\lambda_{j}\left\|I_{t}(\mathbf{x})-b_{t}(\mathbf{x}) c_{t, j}\right\|^{2}\right)^{\frac{1}{1-q}}} \quad i=1,2, \ldots, N .
$$

For the case $q=1$, it can be shown that the minimizer $\hat{u}_{t, i}(\mathbf{x})$ is given by

$$
\hat{u}_{t, i}(\mathbf{x})=\left\{\begin{array}{cl}
1, & i=i_{\text {min }}(\mathbf{x}, t) \\
0, & i \neq i_{\min }(\mathbf{x}, t)
\end{array} \quad i=1,2, \ldots, N\right.
$$

where

$$
i_{\min }(\mathbf{x}, t)=\underset{i}{\operatorname{argmin}}\left(\lambda_{i}\left\|I_{t}(\mathbf{x})-b_{t}(\mathbf{x}) c_{t, i}\right\|^{2}\right)
$$

\subsection{Intensity Similarity and Its Normalization}

The additive noise $n_{t}$ in the image model shown in Eq. (1) should be removed or reduced at least to enhance the accuracy of brain segmentation. There are two drawbacks if the longitudinal images are directly de-noised for each time point separately before performing segmentation. Firstly, excessive bright and/or dark pixels, which may be caused by a particularly strong bias field or the noise, will change image contrast and therefore affect performance of de-noising methods. Secondly, correlations of the longitudinal MR image series in temporal dimension are not taken into account.

Similarly to reference [38], we define

$$
\gamma_{t, i}(\mathbf{x})=\left\|I_{t}(\mathbf{x})-b_{t}(\mathbf{x}) c_{t, i}\right\|^{2}
$$


and use $\gamma_{t}$, defined by $\gamma_{t}=\left(\gamma_{t, 1}, \gamma_{t, 2}, \ldots, \gamma_{t, N}\right)$, to remove the influence of noise on segmentation accuracy. In fact, the vector variable $\gamma_{t}$ can be seen as a similarity vector in which each scalar element $\gamma_{t, i}(\mathbf{x})$ is the intensity similarity between the intensity constant $c_{t, i}$ of the $i$-th tissue and the intensity of the MR image $I_{t}$ at location $\mathbf{x}$

Substituting the above equation into Eq. (11), we obtain

$$
\hat{u}_{t, i}(\mathbf{x})=\frac{\left(\lambda_{i} \gamma_{t, i}(\mathbf{x})\right)^{\frac{1}{1-q}}}{\sum_{j=1}^{N}\left(\lambda_{j} \gamma_{t, j}(\mathbf{x})\right)^{\frac{1}{1-q}}} \quad i=1,2, \ldots, N .
$$

From the above equation, we can see that the membership function $u_{t, i}(\mathbf{x})$ is directly determined by $\gamma_{t, i}(\mathbf{x})$ and the sum of $\gamma_{t, 1}, \gamma_{t, 2}, \ldots$, and $\gamma_{t, N}$. Since fuzzy segmentation results of the image are determined by the membership function $u_{t, i}(\mathbf{x})$ especially by the ratio between different $u_{i}(x, t), i=1,2, \ldots, N$. That is to say final segmentation results are indirectly decided by the ratio between different components of $\gamma_{t}$. As shown in the first row of Fig. 2, the contrasts of intensity similarity images for GM and WM are a little bad. We normalize $\gamma_{t}=\left(\gamma_{t, 1}, \gamma_{t, 2}, \ldots, \gamma_{t, N}\right)$ and obtain the normalization result $\tilde{\gamma}_{t}=\left(\tilde{\gamma}_{t, 1}, \tilde{\gamma}_{t, 2}, \ldots, \tilde{\gamma}_{t, N}\right)$ defined by

$$
\tilde{\gamma}_{t, i}(\mathbf{x})=\frac{\gamma_{t, i}(\mathbf{x})}{\sum_{j=1}^{N} \gamma_{t, j}(\mathbf{x})} \quad i=1,2,3, \ldots, N
$$

It is obvious that this is an inter-class normalization for each image voxel $\mathbf{x}$. Thus, the negative influence of terrible image contrast on noise removal, which is viewed as the first drawback of directly de-noising images separately before segmentation as mentioned earlier, can be overcome. The second drawback can be overcome by the non-locally regularization described in the following subsection. Note that the contrasts of intensity similarity images can also be improved as shown in the second row of Fig. 2.

\subsection{Non-locally Spatio-temporal Regularization by Non-local Means Denoising}

The optimal membership function $\hat{u}_{t, i}(\mathbf{x})$ in Eq. (15) might be irregular due to the noise in the image. In order to accurately segment images described in Eq. (1), effective noise reduction is necessary. However, traditional denoising methods may blur the image especially at boundaries because they generally replace intensity of a given pixel with an average of its neighbouring pixels, which therefore increases the difficulty of segmenting the image correctly [39]. In fact, there is no reason for the most similar pixels to a given pixel to be close to it [40]. Thus, Buades et al. proposed a non-local means denoising (NLMD) method to reduce noise from noisy images by averaging similar pixels in the whole image domain not only in the neighbourhood of a given pixel, which reserves local characteristics of the image and will not blur the image [41].

In order to ensure the consistency of the segmentation, we first define

$$
\left(G_{\delta} *\left\|\tilde{\gamma}_{t}(\mathbf{x}+\cdot)-\tilde{\gamma}_{t}(\mathbf{y}+\cdot)\right\|^{2}\right)(0)=\int_{\Omega} G_{\delta}(\mathbf{s})\left(\sum_{i=1}^{N}\left\|\tilde{\gamma}_{t, i}(\mathbf{x}+\mathbf{s})-\tilde{\gamma}_{t, i}(\mathbf{y}+\mathbf{s})\right\|^{2}\right) d \mathbf{s} .
$$


We then define a non-locally spatio regularization on the components of similarity vector $\tilde{\gamma}_{t}$ as follows

$$
\hat{\tilde{\gamma}}_{t, i}(\mathbf{x})=N L\left(\tilde{\gamma}_{t, i}\right)(\mathbf{x})=\frac{1}{C(\mathbf{x})} \int_{\Omega} \tilde{W}(\mathbf{x}, \mathbf{y}) \tilde{\gamma}_{t, i}(\mathbf{y}) d \mathbf{y}, \quad i=1,2, \ldots, N
$$

where $C(\mathbf{x})=\int \tilde{W}(\mathbf{x}, \mathbf{z}) d \mathbf{z}$ and

$$
\tilde{W}(\mathbf{x}, \mathbf{y})=e^{\frac{-\left(G_{\delta}^{*}\left|\tilde{\gamma}_{t}(\mathbf{x}+.)-\tilde{\gamma}_{t}(\mathbf{y}+.)\right|^{2}\right)(0)}{h^{2}}} .
$$

Eq. (18) indicates that $\tilde{\gamma}_{t, i}(\mathbf{x})$ is replaced by a weighted average of $\tilde{\gamma}_{t, i}(\mathbf{y})$. The weightings are effective only if intensities in the Gaussian window around $\mathbf{y}$ are similar to those in the corresponding window around $\mathbf{x}$. Thus, NLMD takes advantage of image self-similarity to reduce noise and therefore the local characteristics of the image can be preserved as shown in Fig. 2. Differences of intensity similarity between corresponding voxels in the Gaussian windows around $\mathbf{x}$ and $\mathbf{y}$ are summed together for all clusters to ensure a spatio regularization.

To regularize intensity similarity $\tilde{\gamma}_{t, i}$ temporally, we first define

$$
\left(G_{\delta} *\|\tilde{\boldsymbol{\gamma}}(\mathbf{x}+\cdot)-\tilde{\gamma}(\mathbf{y}+\cdot)\|^{2}\right)(0)=\int_{\Omega} G_{\delta}(\mathbf{s})\left(\int_{t=1}^{T} \sum_{i=1}^{N}\left|\tilde{\gamma}_{t, i}(\mathbf{x}+\mathbf{s})-\tilde{\gamma}_{t, i}(\mathbf{y}+\mathbf{s})\right|^{2}\right) d \mathbf{s}
$$

and then rewrite Eq. (19) into

$$
\tilde{W}(\mathbf{x}, \mathbf{y})=e^{\frac{-\left(G_{\delta} * \tilde{\gamma}(\mathbf{x}+.)-\left.\tilde{\gamma}(\mathbf{y}+.)\right|^{2}\right)(0)}{h^{2}}}
$$

and then substitute it into Eq. (18). It is obvious that the time integral in Eq. (20) can be considered as a temporal regularization on the intensity similarities. The regularization is used to overcome the second drawback of directly de-noising images separately as mentioned earlier. We finally write $\hat{\tilde{\gamma}}_{t, i}(\mathbf{x}), i=1,2, \ldots, N$ into a vector form expressed by $\hat{\tilde{\gamma}}_{t}(\mathbf{x})=\left(\hat{\tilde{\gamma}}_{t, 1}(\mathbf{x}), \hat{\tilde{\gamma}}_{t, 2}(\mathbf{x}), \ldots, \hat{\tilde{\gamma}}_{t, N}(\mathbf{x})\right)^{T}$.

\subsection{Non-locally Regularized Membership Function}

The membership functions determined by the de-noised intensity similarities in Eq.(18) can be denoted by $\hat{\tilde{\mathbf{u}}}_{t}(\mathbf{x})=\left(\hat{\tilde{u}}_{t, 1}(\mathbf{x}), \hat{\tilde{u}}_{t, 2}(\mathbf{x}), \ldots, \hat{\tilde{u}}_{t, N}(\mathbf{x})\right)^{T}$. For $q>1$, each component of $\hat{\tilde{\mathbf{u}}}_{t}(\mathbf{x})$ is given by

$$
\hat{\tilde{u}}_{t, i}(\mathbf{x})=\frac{\left(\lambda_{i} \hat{\tilde{\gamma}}_{t, i}(\mathbf{x})\right)^{\frac{1}{1-q}}}{\sum_{j=1}^{N}\left(\lambda_{j} \hat{\tilde{\gamma}}_{t, j}(\mathbf{x})\right)^{\frac{1}{1-q}}} \quad i=1,2, \ldots, N .
$$

${ }_{187}$ For $q=1$, it can be shown that

$$
\hat{\tilde{u}}_{t, i}(\mathbf{x})=\left\{\begin{array}{ll}
1, & i=i_{\text {min_reg }}(\mathbf{x}, t) \\
0, & i \neq i_{\text {min_reg }}(\mathbf{x}, t)
\end{array} \quad i=1,2, \ldots, N\right.
$$

188 where

$$
i_{\text {min_reg }}(\mathbf{x}, t)=\underset{i}{\arg \min }\left(\lambda_{i} \hat{\tilde{\gamma}}_{t, i}(\mathbf{x})\right)
$$



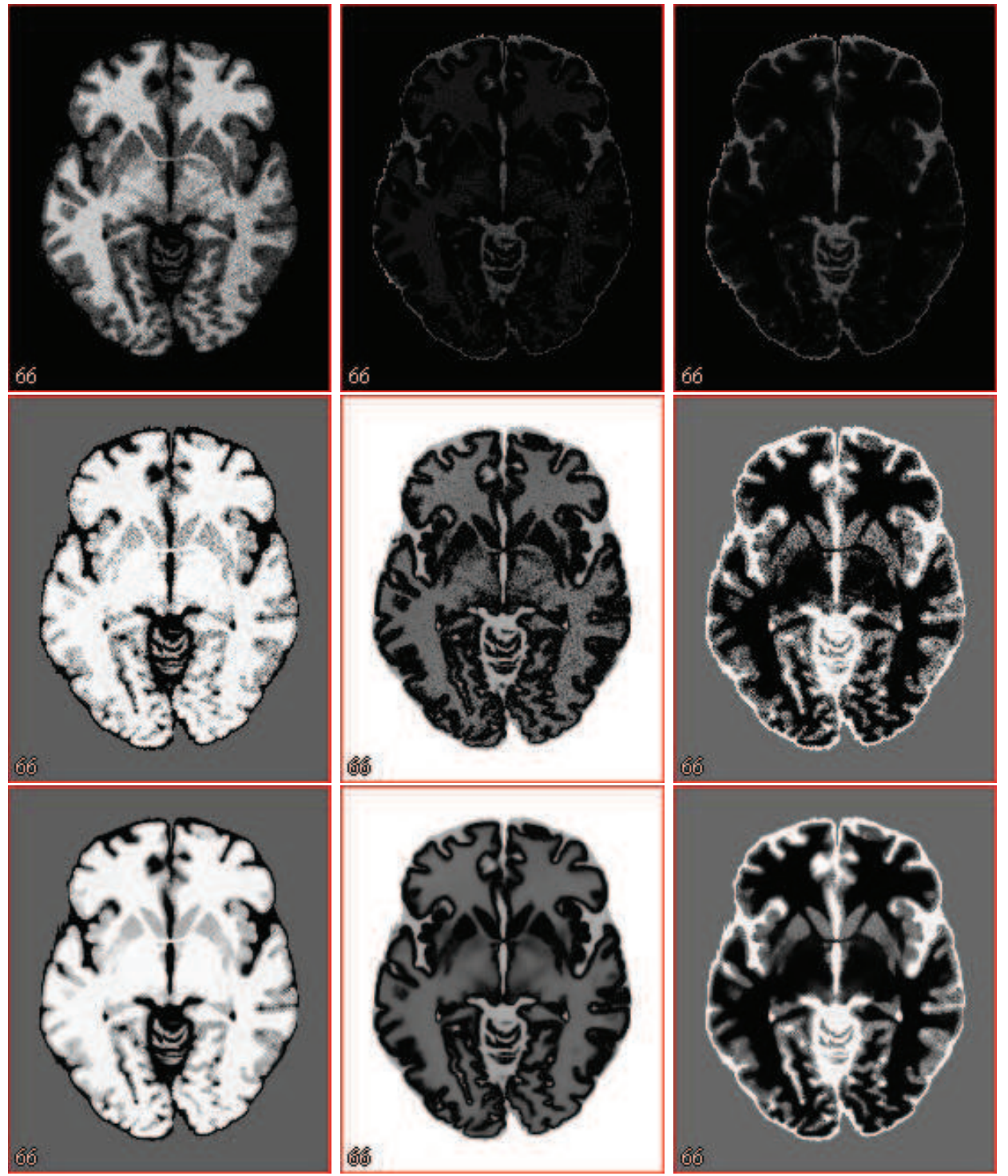

Figure 2: Demonstration of effect of the proposed non-locally spatio-temporal regularization on one brain MR image from a simulated patient case. The intensity similarities computed by Eq. (14), normalized results in Eq. (16), and final regularized results obtain from Eq. (18) are given in the 1st, 2nd, and 3rd rows, respectively. Images in each row are results computed for CSF, GM, WM from left to right, respectively. 


\subsection{Implementation}

To ensure the slowly and smoothly varying property of the bias filed, we convolute the bias field $b$ with a kernel function $K$ in the implementation to smooth it as soon as it has been updated using Eq. (9) in each iteration. Note that the choice of the convolution kernel $K$ is important but flexible as long as it is a non-negative function defined on $\Omega$ with range of $[0,+\infty)]$ and satisfies:

1) $K(-\mathbf{x})=K(\mathbf{x})$;

2) $K(\mathbf{x})>K(\mathbf{y})$, if $|\mathbf{x}|<|\mathbf{y}|$, and $\lim _{|\mathbf{x}| \rightarrow+\infty} K(\mathbf{x})=0$;

3) $\int_{\Omega} K(\mathbf{x}) d \mathbf{x}=1$.

In this paper, the following averaging filter with size of $\rho$ is chosen as the convolution kernel function $K$ :

$$
K_{\rho}(\mathbf{u})=\left\{\begin{array}{lll}
a, & \text { for } & |\mathbf{u}| \leq \rho \\
0, & \text { for } & |\mathbf{u}|>\rho
\end{array}\right.
$$

where $a>0$ is a normalization factor such that $\int_{|\mathbf{u}| \leq \rho} K_{\rho}(\mathbf{u})=1$.

The implementation of the proposed method can be straightforwardly expressed in the following steps.

- Step 1. Set $t=1$. For images at time point $t$, initialize $\mathbf{u}_{t}(\mathbf{x})$ and $\mathbf{c}_{t}$.

- Step 2. Update $b_{t}(\mathbf{x})$ as $\hat{b}_{t}(\mathbf{x})$ computed by Eq.(9).

- Step 3. Convolute $b_{t}(\mathbf{x})$ with kernel $K$ to smooth it.

- Step 4. Update $\mathbf{c}_{t}$ as $\hat{\mathbf{c}}_{t}$ with $\hat{c}_{t, i}$ computed by Eq.(8).

- Step 5. Update $\mathbf{u}_{t}(\mathbf{x})$ as $\hat{\mathbf{u}}_{t}(\mathbf{x})$ with $\hat{u}_{t, i}(\mathbf{x})$ computed by Eq.(11) for $q>1$ or Eq.(12) for $q=1$.

- Step 6. Go to Step 2 unless objective function defined in Eq. (7) converged or the iteration exceeds a predetermined maximum number.

- Step 7. Generate $\gamma_{t}(\mathbf{x})$ with $\gamma_{t, i}(\mathbf{x})$ computed by Eq.(14).

- Step 8. Normalize $\gamma_{t}(\mathbf{x})$ to generate $\tilde{\gamma}_{t}(\mathbf{x})$ with its component $\tilde{\gamma}_{t, i}(\mathbf{x})$ computed by Eq.(16).

- Step 9. Set $t=t+1$. If $t \leq T$, go to Step 1. Otherwise, go to Step 9 .

- Step 10. For each time point $t \in[1, T]$, generate $\hat{\tilde{\gamma}}_{t}(\mathbf{x})$ with its component $\hat{\tilde{\gamma}}_{t, i}(\mathbf{x})$ computed by Eq. (18).

- Step 11. Update the membership function $\hat{\tilde{\mathbf{u}}}_{t}(\mathbf{x})$ with its component $\hat{\tilde{u}}_{t, i}(\mathbf{x})$ computed by Eq. $(22)$ for $q>1$ or by Eq.(23) for $q=1$.

The convergence criterion used in Step 5 is $\left\|\mathbf{c}_{t}^{(n+1)}-\mathbf{c}_{t}^{(n)}\right\|<0.003$, where $\mathbf{c}_{t}^{(n+1)}$ and $\mathbf{c}_{t}^{(n)}$ are the updated vector $\mathbf{c}_{t}$ in Step 3 in the $n+1$-th and $n$-th iteration, respectively. Since the images are classified into WM, GM, and CSF, we set $N=3$ in this paper. We respectively set other parameters $\lambda_{1}=1.7$, $\lambda_{2}=1.0, \lambda_{3}=0.8, q=2.0$, and $\rho=7$ as the default parameters for segmenting WM, GM, and CSF from the images.

The original definition of the NL-means algorithm considers that each voxel can be linked to all the others, but the number of voxels taken into account in the weighted average is practically restricted in a neighborhood of size $(2 M+1)^{3}$ centered at current voxel $i$. This is the so called search window where 
the similarity between $i$ and $j$ depends on the similarity of their local neighbourhoods of size $(2 d+1)^{3}$. Therefore, the main disadvantage of the NL-means algorithm is the computational burden as its complexity is in the order of $O\left(\left(N(2 M+1)^{3}(2 d+1)^{3}\right)\right)$ for images with $N$ voxels. As reported in [42], for the image with resolution of $181 \times 217 \times 181$, the computational time reaches up to 6 hours for NLMD with parameters $d=1$ and $M=5$. This is far beyond a reasonable duration expected by a de-noising algorithm in medical practice. As improvement of GPUs in the capability of parallel programming is remarkable in the last few years [43], we accelerate the modified de-noising described in Eq.(18) using GPUs in the implementation. The GPUs with Compute Unified Device Architecture (CUDA) are treated as data-parallel computation devices [44], in which thousands of threads simultaneously execute at the same time [45]. By using standard C language, the modified NLMD algorithm can be easily implemented by encapsulating its instructions into a CUDA kernel function. The function is invoked by the CPU but executed on the GPU with thousands of threads concurrently. Unfortunately, there is a watchdog timer to trigger a system reset if the kernel occupies the GPU longer than 5-15 seconds depending on the specific setting of the operating system. Readers are referred to http://msdn.microsoft.com/en-us/library/ms856964 for more details. Therefore, in our implementation, CUDA threads are created based on the resolution of the input image. If the watchdog time reaches for larger search window and/or neighbourhood, the kernel function is split into $(2 M+1)^{3}$ smaller functions which will introduce $(2 M+1)^{3}$ times launch and invocation but short the execution time for each thread. If the watchdog time reaches again (hardly happen), the kernel functions are further split into $(2 d+1)^{3}$ more smaller kernels and will introduce $(2 M+1)^{3} *(2 d+1)^{3}$ times launch and invocation. In this way, we obtain about 200 times speedup for the proposed algorithm implemented on Geforce GT310 (45 seconds)than its running on intel i7 processor (about 2 hours and a half) to de-noise a $181 \times 217 \times 181$ image with $M=2$ and $d=1$. Due to the achievement of watchdog time and $(2 M+1)^{3}$ times launch and invocation, the speedup ratio reduces to 150 (less than 10 minutes) for de-noising a image with resolution of $181 \times 217 \times 181$ in $M=5$ and $d=1$.

\section{Experimental Results}

The proposed method has been extensively tested on both synthetic images and real images in Matlab R2011b on a computer with Intel(R) Core(TM) i5-3230M 2.6 GHz CPU, 4 GB RAM, and Windows 7 64-bit operating system. The synthetic images downloaded from BrainWeb (http://www.bic.mni.mcgill.ca/ brainweb/), which also provides the ground truth that can be used to quantitatively evaluate the accuracy of the proposed segmentation method. We first downloaded 9 cases of MR images with three different levels of noise (none, $5 \%$, and $9 \%$ to the brightest tissue) and three levels of intensity inhomogeneity (none, $20 \%$, and $40 \% \mathrm{RF}$ ) from the website. Resolutions of the images are $181 \times 217 \times 181$ with $1 \mathrm{~mm}$ in-plane pixel size and $1 \mathrm{~mm}$ slice thickness. Note that intensity inhomogeneities in the images are linear and they 
are therefore easily handled by segmentation methods. To examine segmentation methods in a much more difficult situation, we added three levels of non-linear intensity inhomogeneities to the simulated image by multiplying them with the original image downloaded from BrainWeb to obtain three images with different intensity inhomogeneities. We then added noise of three different levels to these images and therefore obtained another 9 cases of MR images. In addition, simulated brain atrophy are added to the 18 MR image series. The real images are from the Internet Brain Segmentation Repository (IBSR) which is a World Wide Web resource providing access to MR brain images and segmentation results contributed and utilized by researchers from all over the world. In this section, we first apply the proposed method BCEFCM to segment Brainweb and IBSR datasets and compare segmentation accuracy of BCEFCM, FCM, and BCFCM on the datasets qualitatively. We then demonstrate the capability of the proposed BCEFCM+NL method in segmentation of brain MR images in arbitrary number of serial images. We finally quantitatively validate segmentation accuracy of the proposed BCEFCM+NL method in segmentation of longitudinal images and its stability.

\subsection{Effectiveness of BCEFCM on segmentation of Brain MR Images}

In this subsection, we validate effectiveness of BCEFCM on segmentation of brain MR images on BrainWeb and IBSR datasets. We first compare segmentation results of BCEFCM with FCM and BCFCM on four image slices from the same image case of BrainWeb with the most severe non-linear intensity inhomogeneity $(60 \%)$ and show the results in Fig. 3. Estimated bias field and bias corrected images are also given in Fig. 3. It can be obviously seen that 1) segmentation results of FCM are the most easily affected by intensity inhomogeneity, 2) BCFCM considered parts of GM as WM, 3) BCEFCM is not sensitive to intensity inhomogeneity with its segmentation results match with the ground truth best, and 4) the estimated bias field of BCEFCM is smooth and intensities of the corrected image are more homogeneous. We then compare segmentation results of BCEFCM with FCM and BCFCM on four image slices from case IBSR_04 of IBSR dataset and show the results in Fig. 4. Estimated bias field and bias corrected images are included as shown in Fig. 4. It can be seen that 1) parts of GM are considered as WM by BCFCM, 2) the amount of CSF and WM are both obviously greater than the ground truth in segmentation results of FCM, 3) segmentation results of BCEFCM match with the ground truth best, and 4) the estimated bias field is smooth and intensities of the corrected image are more homogeneous. All above, the proposed BCEFCM method is effective in segmenting brain MR images and estimating the bias field accurately.

\subsection{Brain Segmentation in Arbitrary Number of Serial MR Images}

It is obvious that the proposed method is able to produce desirable results for brain segmentation even if there are severe intensity inhomogeneities in brain MR images. Furthermore, the non-local means de-noising regularization enables the proposed method to produce noise-free segmentation results. The third advantage 


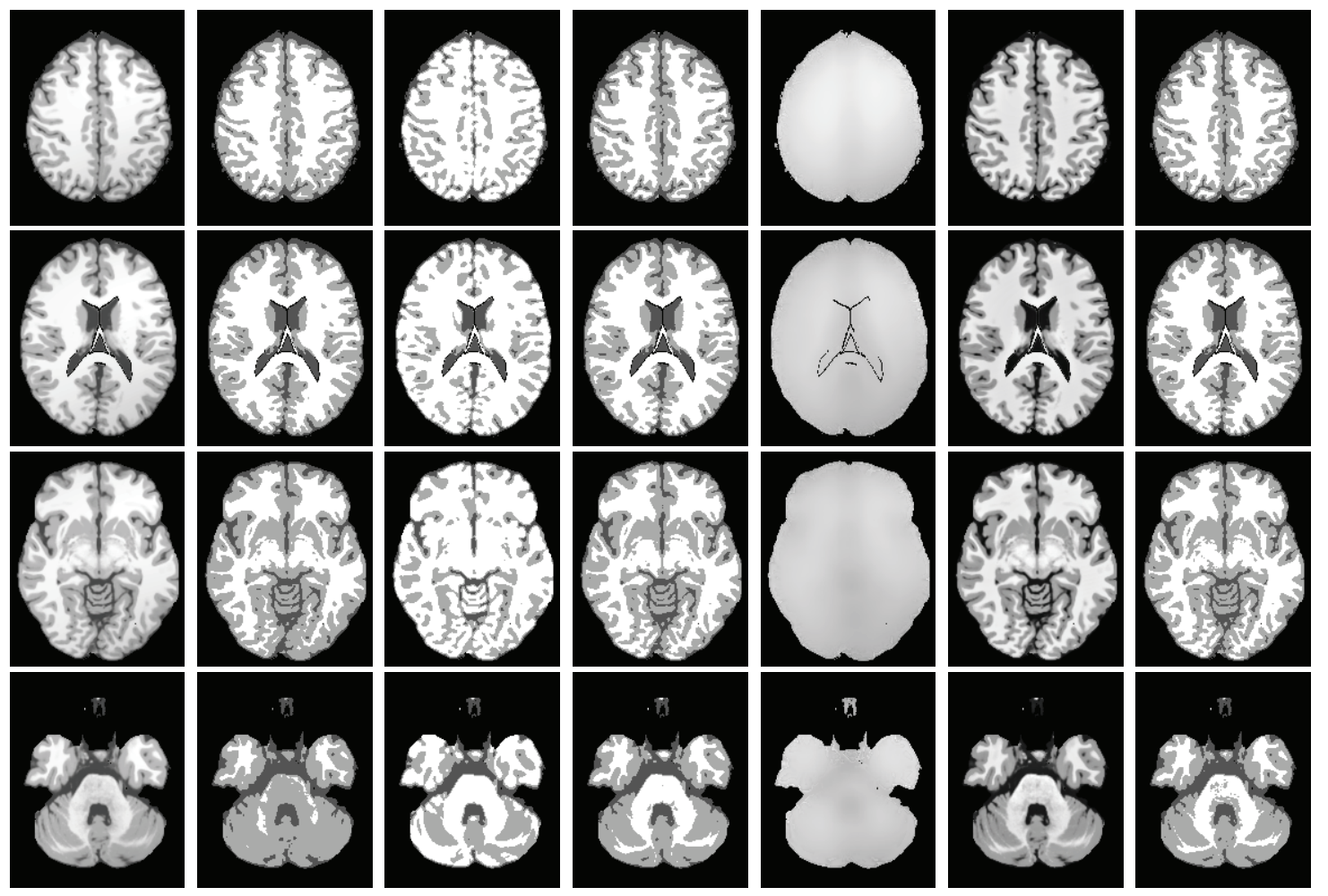

Figure 3: Comparison of segmentation results of BCEFCM with state-of-the-art methods on four slices of one image case from BrainWeb with the most severe non-linear intensity inhomogeneity (60\%). From left to right: original images, segmentation results of FCM, BCFCM, and BCEFCM, estimated bias field and corrected images of BCEFCM, and the ground truth.
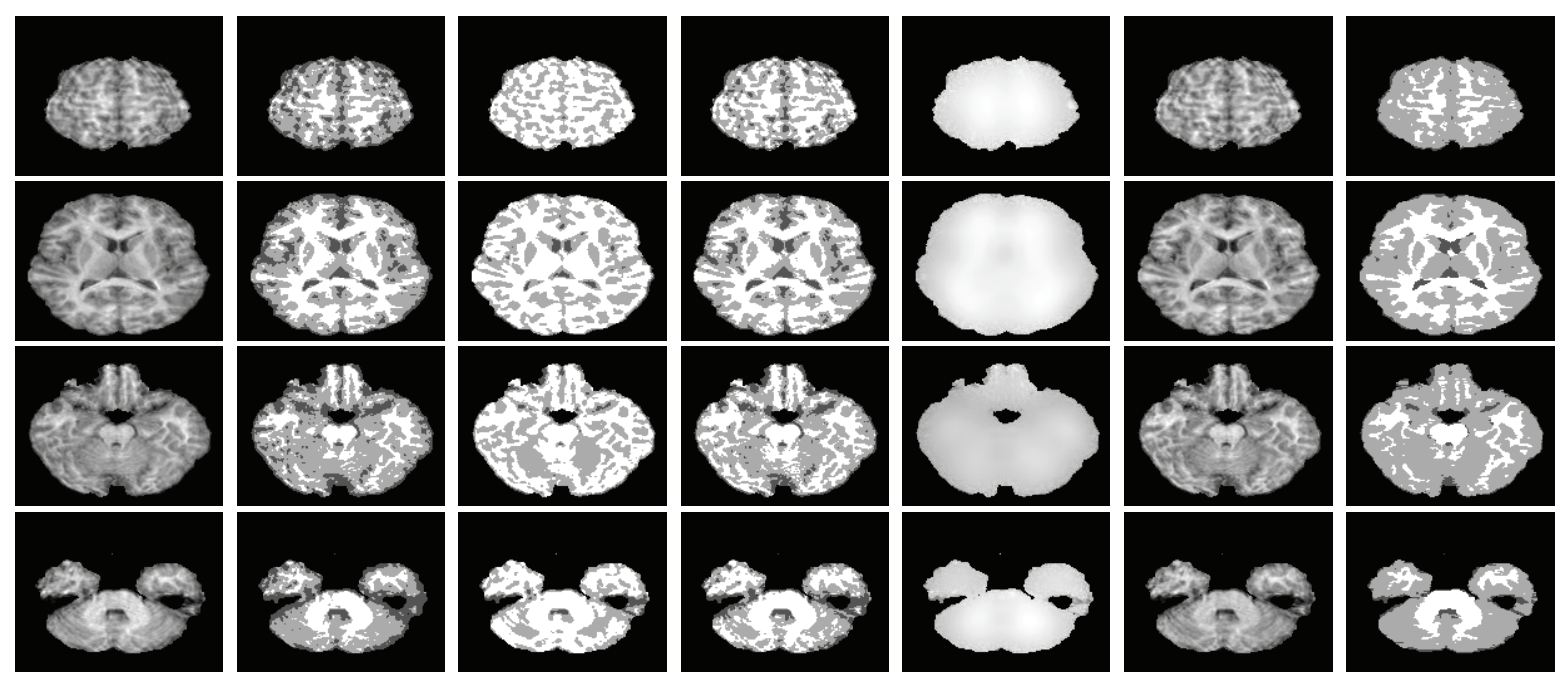

Figure 4: Comparison of segmentation results of BCEFCM with state-of-the-art methods on four slices from case IBSR_04 in four rows. For each slice, the original image, segmentation results of FCM, BCFCM, and BCEFCM, estimated bias field and corrected images of BCEFCM, and the ground truth are given from left to right, respectively. 

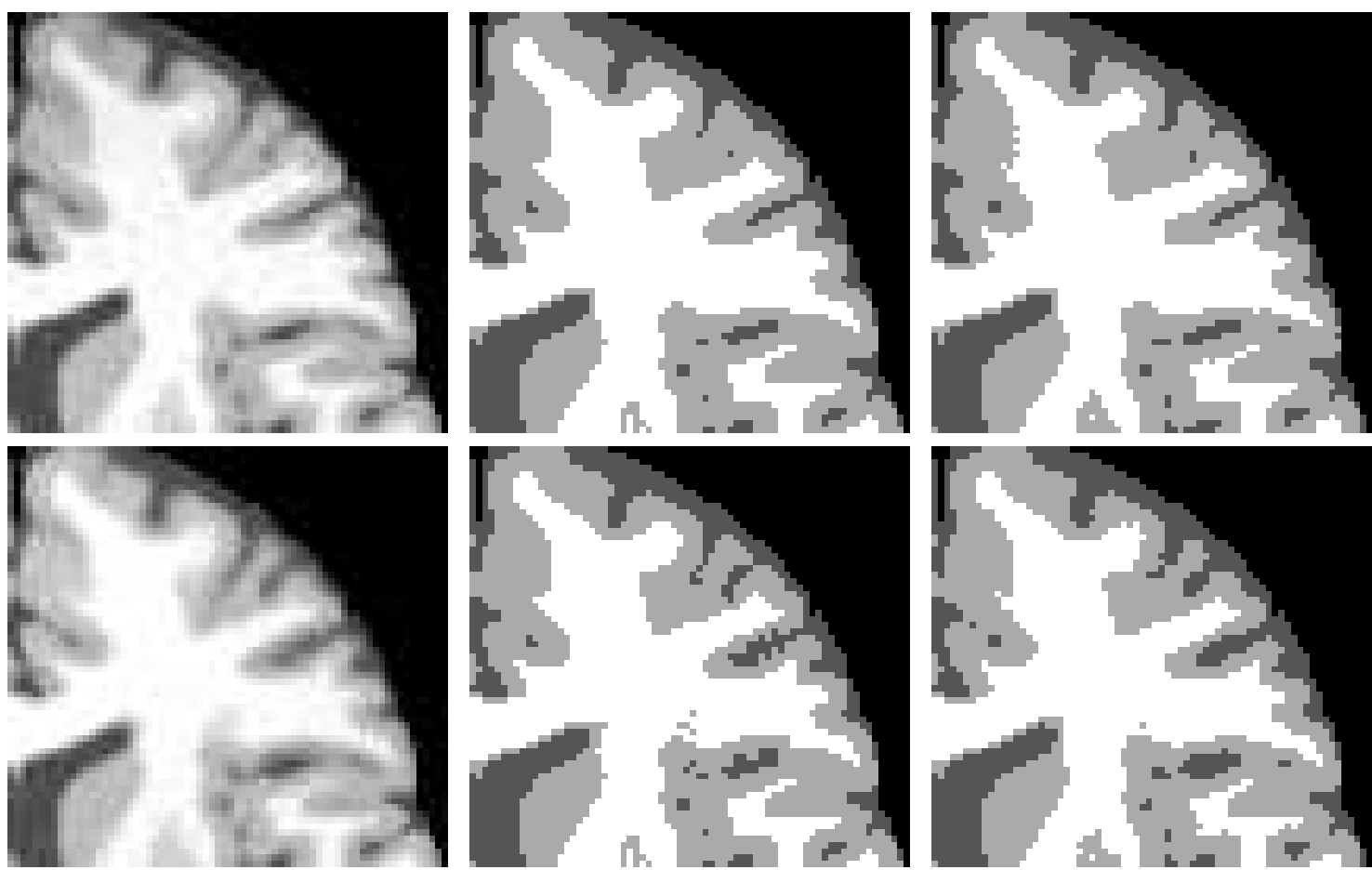

Figure 5: Demonstration of application of the proposed method to segment brain MR image series with simulated atrophy at 2-time points. Images in upper and lower rows are chronological with original images (40\% bias and $5 \%$ noise), the ground truth, and segmentation results of the proposed method shown in the left, middle, and right columns, respectively.

of the proposed method is that it can segment brain MR images scanned in an arbitrary number of time series. To verify this advantage of the proposed method, we applied the proposed method to segment brain MR images at 2-time points and 3-time points and show one slice of segmentation results in Fig.5 and Fig.6, respectively. The images come from one longitudinally synthetic image series with cerebral atrophy. It is obvious that although there are severe intensity inhomogeneities ( $40 \%$ linear bias) and noise (5\% to the brightest tissue) in the MR images, the proposed method can segment brain tissues from the longitudinal MR images no matter how many time series the images include and segmentation results of the proposed method are close to the ground truth.

\subsection{Quantitative Evaluation of Segmentation Accuracy}

To evaluate segmentation accuracy of the proposed method quantitatively, we compare segmentation results of the proposed method with FAST, FreeSurfer and BCEFCM on BrainWeb in terms of false positive ratio (FPR), false negative ratio (FNR), and Jaccard similarity (JS) in this subsection.

For image segmentation, a false positive occurs when a segmentation result indicates that a pixel belongs to a tissue, but it does not in fact. On the contrary, a false negative occurs when the segmentation result indicates that a pixel does not belong to a tissue, but it does in fact. Let NFP and NFN be the number of 

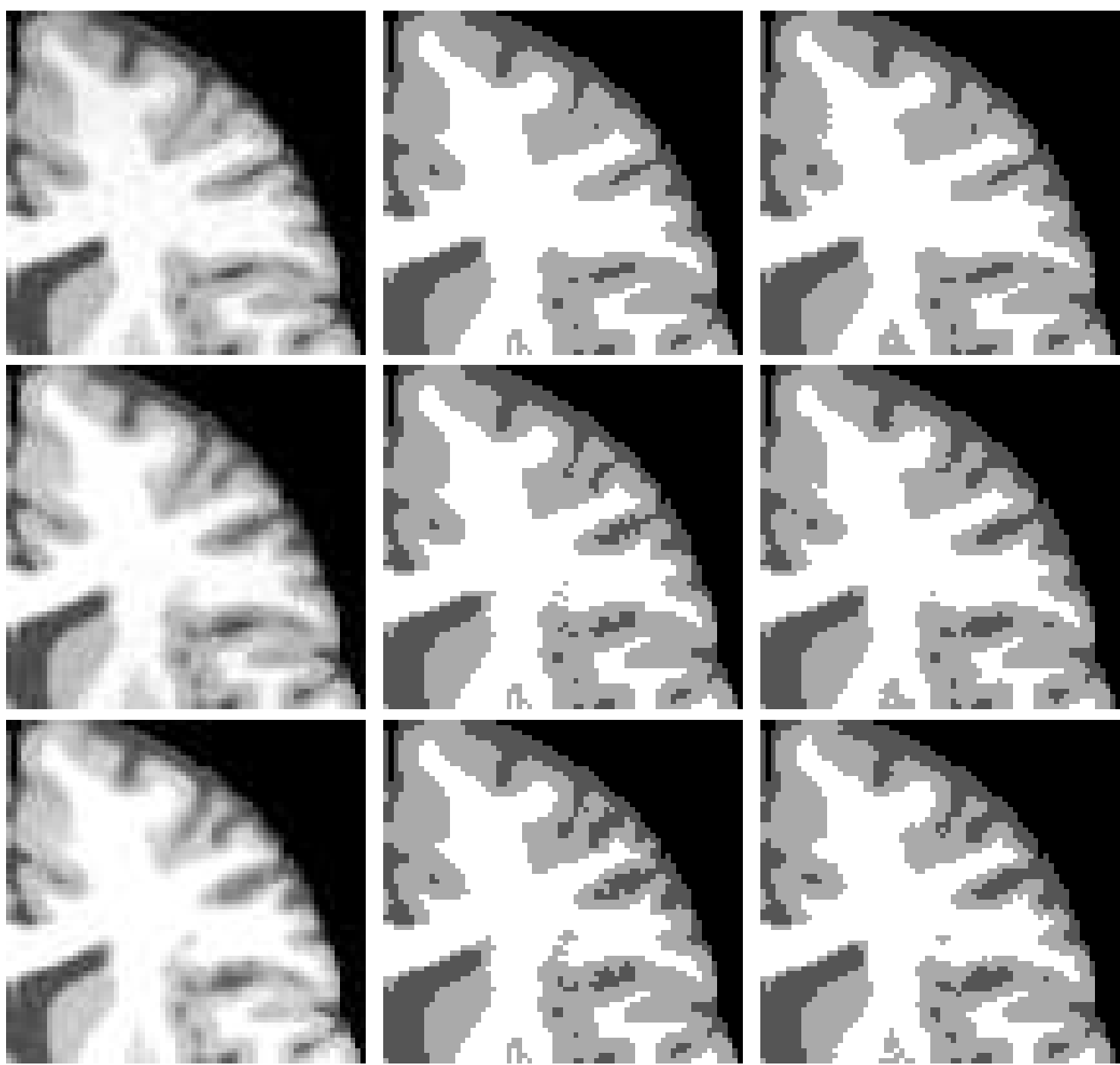

Figure 6: Demonstration of application of the proposed method to segment brain MR image series with simulated atrophy at 3 -time points. Images in upper, middle, and lower rows are chronological with original images (40\% bias and $5 \%$ noise), the ground truth, and segmentation results of the proposed method shown in left, middle, and right columns, respectively. 

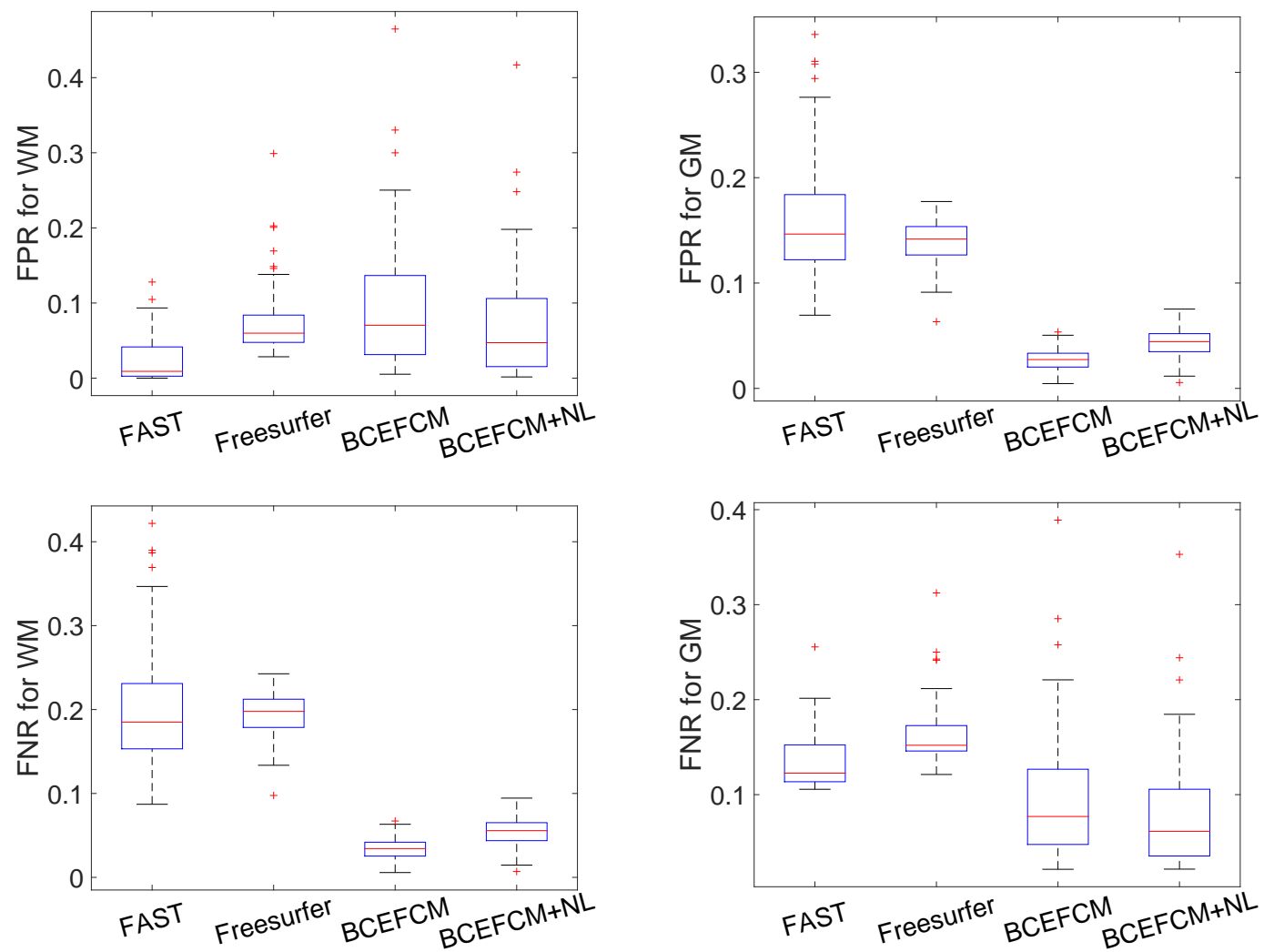

Figure 7: Comparison of the proposed method with FAST, FreeSurfer, and BCEFCM on BrainWeb images in terms of FPR (upper row) and FNR (lower row).

FP and FN and $A$ be the ground truth, FPR and FNR can then be defined by

$$
\mathrm{FPR}=\frac{\mathrm{NFP}}{|I|-|A|} \times 100 \%
$$

and

$$
\mathrm{FNR}=\frac{\mathrm{NFN}}{|A|} \times 100 \%,
$$

respectively, where $|*|$ denotes the size of region $*$. Obviously, values of FPR and FNR both range from 0 and 1 with a smaller value indicating a better match between segmentation result and the ground truth. To evaluate segmentation accuracy of the proposed method quantitatively, we compare FPR and FNR values of segmentation results obtained by the proposed method on BrainWeb images in 3-time point with FAST, FreeSurfer, and BCEFCM and show the comparison result in Fig.7. In this boxplot figure, shorter boxes and lower values indicate a better match between segmentation results and the ground truth. It is obvious that the FNR values of the proposed method for GM are smaller than the other methods. Whereas, FPR values of the proposed method for GM are slightly larger than BCEFCM. For WM, FPR values of the proposed method are slightly larger than FAST and FNR values are slightly larger than BCEFCM. 

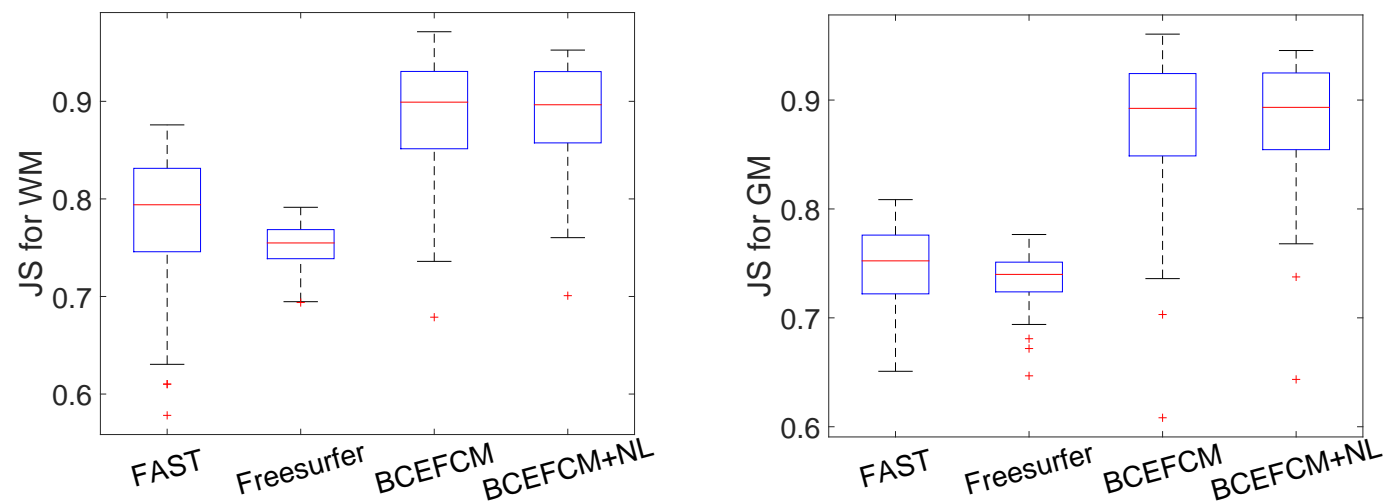

Figure 8: Comparison of the proposed method with FAST, FreeSurfer, and BCEFCM on BrainWeb images in terms of JS.

More objective and precise comparison of segmentation accuracy of the proposed methods with others can performed by evaluating the segmentation results using Jaccard similarity, which is defined as the size of the intersection divided by the size of the union of the segmentation result and the ground truth as described in the following form

$$
J S=\frac{\left|S_{1} \cap S_{2}\right|}{\left|S_{1} \cup S_{2}\right|}
$$

where $|*|$ represents the area of region $*, \cap$ is the intersection operator, $\cup$ is the union operator, $S_{1}$ is the region segmented by an algorithm, and $S_{2}$ is the corresponding region obtained from the ground truth. Obviously, $J S \in[0,1]$ with higher value indicating the segmentation result better match with the ground truth. Segmentation accuracy of FAST, FreeSurfer, BCEFCM, and the proposed method in term of JS on BrainWeb are shown in Fig.8. In this boxplot figure, shorter boxes and higher values indicate a better match between segmentation results and the ground truth. From Fig. 8, we can obviously see that segmentation results of the proposed method are the closest to the ground truth. Specifically, the box range and the band in the box (median) of the proposed method are almost equal to BCEFCM and greater than other methods. Although the maximal JS for the proposed method is less than BCEFCM, the minimum of the proposed method is the largest.

\subsection{Stability of the Proposed Method for Longitudinal Segmentation}

As mentioned earlier, the proposed method is effective for segmenting brain tissues in an arbitrary number of brain MR image series. Thus, there is no restriction on the number of image series for the proposed method to achieve a longitudinal segmentation. No matter how many time series $(2$, 3 , or more) the longitudinal images include, the proposed method can provide a stably longitudinal segmentation. We evaluate segmentation stability of the proposed method on longitudinal images from BrainWeb in this section. To give a statistical analysis, the volumes of GM and WM marked by the proposed method, ground truth, and FAST, FreeSurfer, and BCEFCM on the 30 BrainWeb image series are first computed. The 

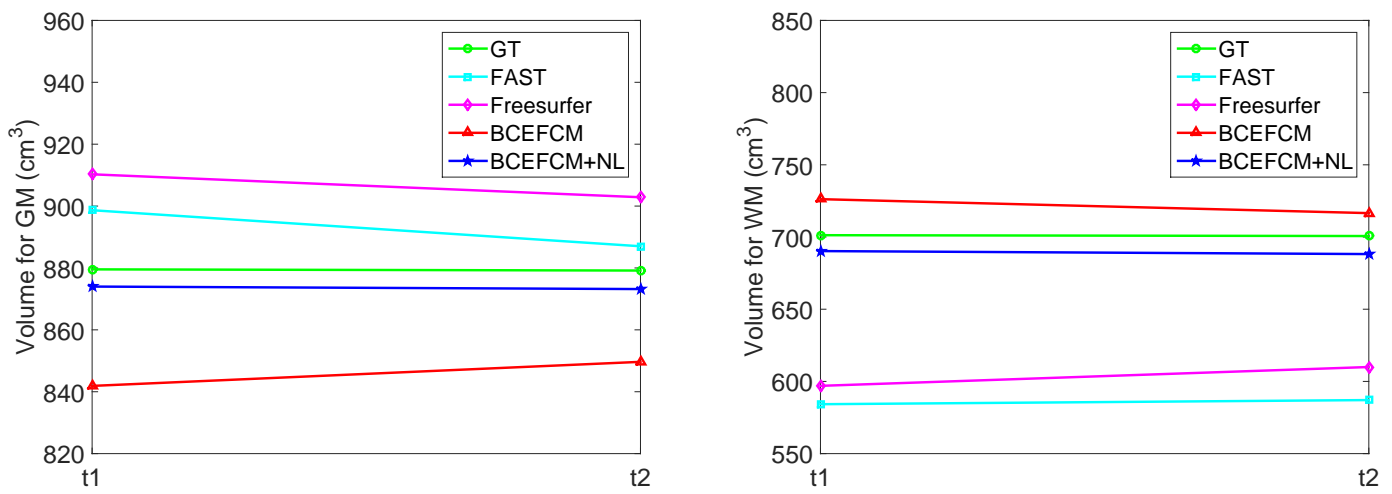

Figure 9: Comparison of segmentation consistency of the proposed method with the ground truth (GT), FAST, FreeSurfer, and BCEFCM in terms of volume change for 2-time point images from BrainWeb.
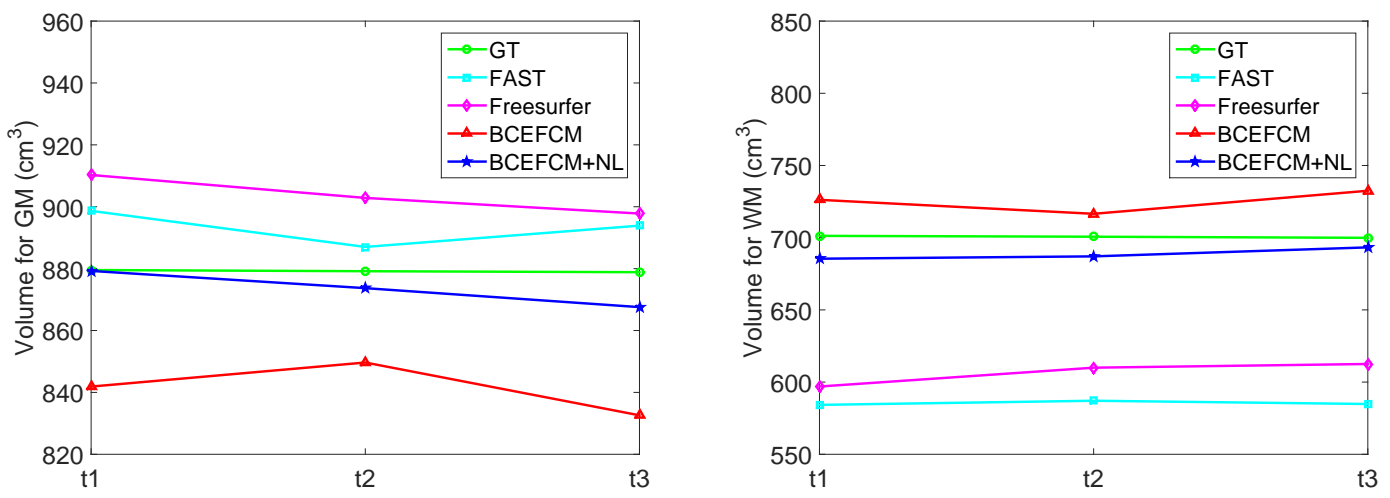

Figure 10: Comparison of segmentation consistency the proposed method with the ground truth (GT), FAST, FreeSurfer, and BCEFCM in terms of volume change for 3-time point images from BrainWeb.

medians are then extracted and plotted in Fig.9 and Fig.10 for 2-time point segmentation and 3-time point segmentation, respectively. For the segmentation methods that are not effective for segmenting images in 2- or 3- time points, we segment the entire longitudinal image series using them separately and extract segmentation results of the corresponding series used in this comparison to analyse their performance in volume estimation of GM and WM. As shown in Fig.9 and Fig.10, the trends of volume curves of GM and WM computed from segmentation results of the proposed method give the best match with the ground truth and indicate brain atrophy well. 


\section{Discussions}

\subsection{Impact of Weighting Coefficients $\lambda_{i}$}

Let $n=\frac{1}{q-1}$, Eq.(15) can be rewritten as

$$
\hat{u}_{t, i}(\mathbf{x})=\frac{1}{\sum_{j=1}^{i-1}\left(\frac{\lambda_{i} \gamma_{t, i}(\mathbf{x})}{\lambda_{j} \gamma_{t, j}(\mathbf{x})}\right)^{n}+\sum_{j=i+1}^{N}\left(\frac{\lambda_{i} \gamma_{t, i}(\mathbf{x})}{\lambda_{j} \gamma_{t, j}(\mathbf{x})}\right)^{n}+1} .
$$

If $\Delta \lambda_{i}>0$, we substitute $\lambda_{i}^{\prime}=\lambda_{i}+\Delta \lambda_{i}$ into the above equation and obtain

$$
\hat{u}_{t, i}^{\prime}(\mathbf{x})=\frac{1}{\sum_{j=1}^{i-1}\left(\frac{\left(\lambda_{i}+\Delta \lambda_{i}\right) \gamma_{t, i}(\mathbf{x})}{\lambda_{j} \gamma_{t, j}(\mathbf{x})}\right)^{n}+\sum_{j=i+1}^{N}\left(\frac{\left(\lambda_{i}+\Delta \lambda_{i}\right) \gamma_{t, i}(\mathbf{x})}{\lambda_{j} \gamma_{t, j}(\mathbf{x})}\right)^{n}+1} .
$$

Thus, difference between $\hat{u}_{t, i}^{\prime}(\mathbf{x})$ and $\hat{u}_{t, i}(\mathbf{x})$ can be written as

$$
\begin{aligned}
\Delta \hat{u}_{t, i}(\mathbf{x}) & =\hat{u}_{t, i}^{\prime}(\mathbf{x})-\hat{u}_{t, i}(\mathbf{x}) \\
& =\frac{\sum_{j=1}^{i-1}\left(\left(\frac{\lambda_{i} \gamma_{t, i}(\mathbf{x})}{\lambda_{j} \gamma_{t, j}(\mathbf{x})}\right)^{n}-\left(\frac{\left(\lambda_{i}+\Delta \lambda_{i}\right) \gamma_{t, i}(\mathbf{x})}{\lambda_{j} \gamma_{t, j}(\mathbf{x})}\right)^{n}\right)+\sum_{j=i+1}^{N}\left(\left(\frac{\lambda_{i} \gamma_{t, i}(\mathbf{x})}{\lambda_{j} \gamma_{t, j}(\mathbf{x})}\right)^{n}-\left(\frac{\left(\lambda_{i}+\Delta \lambda_{i}\right) \gamma_{t, i}(\mathbf{x})}{\lambda_{j} \gamma_{t, j}(\mathbf{x})}\right)^{n}\right)}{\left(\sum_{j=1}^{N}\left(\frac{\lambda_{i} \gamma_{t, i}(\mathbf{x})}{\lambda_{j} \gamma_{t, j}(\mathbf{x})}\right)^{n}\right)\left(\sum_{j=1}^{N}\left(\frac{\left(\lambda_{i}+\Delta \lambda_{i}\right) \gamma_{t, i}(\mathbf{x})}{\left(\lambda_{j}+\Delta \lambda_{j}\right) \gamma_{t, j}(\mathbf{x})}\right)^{n}\right)}
\end{aligned}
$$

It is obvious that $\frac{\left(\lambda_{i}+\Delta \lambda_{i}\right) \gamma_{t, i}(\mathbf{x})}{\lambda_{j} \gamma_{t, j}(\mathbf{x})}>\frac{\lambda_{i} \gamma_{t, i}(\mathbf{x})}{\lambda_{j} \gamma_{t, j}(\mathbf{x})}>0$. It is well known that the power function $x^{n}$ is positive and is monotonically increasing in the interval of $(0, \infty)$ if $n>0$. Thus, $\left(\frac{\left(\lambda_{i}+\Delta \lambda_{i}\right) \gamma_{t, i}(\mathbf{x})}{\lambda_{j} \gamma_{t, j}(\mathbf{x})}\right)^{n}>\left(\frac{\lambda_{i} \gamma_{t, i}(\mathbf{x})}{\lambda_{j} \gamma_{t, j}(\mathbf{x})}\right)^{n}$. Therefore, for the case $q>1, \Delta \hat{u}_{i}(\mathbf{x})<0$ if $\Delta \lambda_{i}>0$. For the case $q=1$, it can be seen obviously from Eq.(12) that the increase of $\lambda_{i}$ will decrease the possibility of $\operatorname{argmin}_{i}\left(\lambda_{i} \gamma_{t, i}\right)$ being $i$. That is to say, $\hat{u}_{t, i}(\mathbf{x})$ can been viewed as a monotonic decreasing function of $\lambda_{i}$ for $q \geq 1$, which indicates that a greater $\lambda_{i}$ will result in reduction of the amount of pixels classified as the $i$-th tissue. To demonstrate the impact of $\lambda_{i}$ visually, we segment the 128-th image slice of IBSR_09 with nine different values of $\lambda_{2}$ equally spaced in the interval of $[0.4,2.0]$ where $\lambda_{1}$ and $\lambda_{3}$ are both set to be 2.0 and show the results in Fig. 11. In this experiment, cluster centroids are initialized as equally spaced values in the range of intensities for the image. It can be seen from the original image as shown in Fig. 11(a), the intensities of GM are lower than WM but greater than CSF. Therefore, the increase of $\lambda_{2}$ will decrease the amount of pixels classified as GM as shown in Fig. 11(b), which can be also seen visually from GM segmented by BCEFCM with $\lambda_{2}$ changing with step 0.2 in the interval of $[0.4,2.0]$ as shown in Fig. 11(c) to Fig. 11(k).

\subsection{Impact of Parameter $\rho$}

As mentioned earlier, the bias field is smoothed once it is updated in each iteration by convoluting it with a kernel and the averaging filter $K_{\rho}$ is used in this paper. Note that the size (element number) of convolution kernel $K_{\rho}$ is controlled by the parameter $\rho$. In fact, the greater the parameter $\rho$ is, the larger the scale of $K_{\rho}$ is and the smoother the estimated bias field is. To demonstrate the impact of $\rho$ on the smoothness of the estimated bias field and on the quality of the corrected image visually, we segment the 


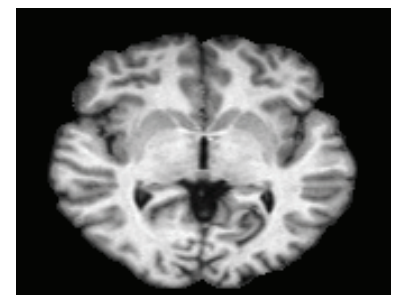

(a) The original image

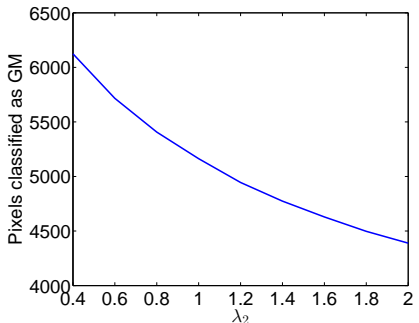

(b) The amount curve of GM

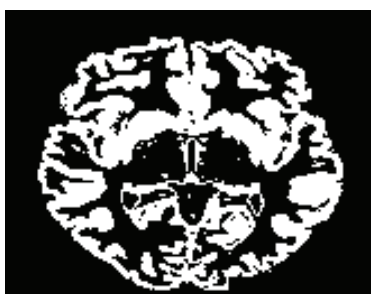

(c) $\lambda_{2}=0.4$

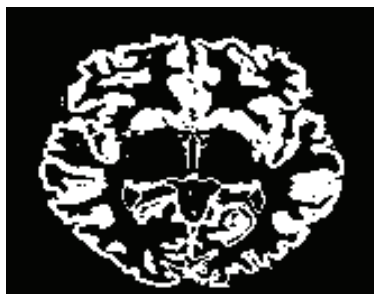

(f) $\lambda_{2}=1.0$

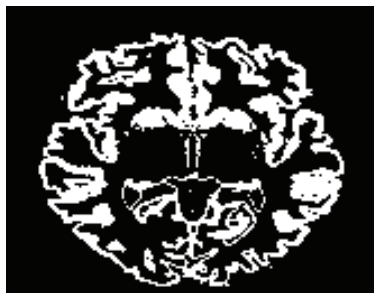

(i) $\lambda_{2}=1.6$

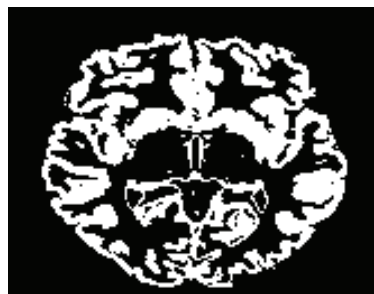

(d) $\lambda_{2}=0.6$

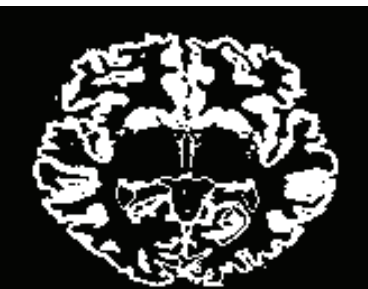

(g) $\lambda_{2}=1.2$

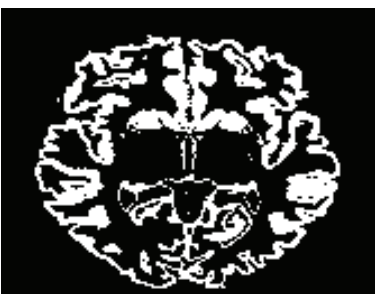

(j) $\lambda_{2}=1.8$

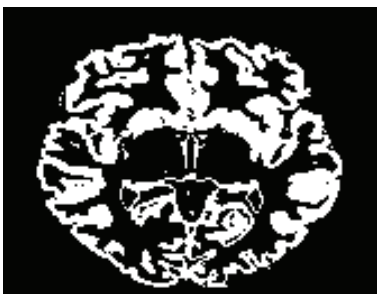

(e) $\lambda_{2}=0.8$

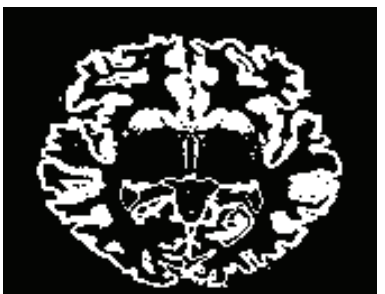

(h) $\lambda_{2}=1.4$

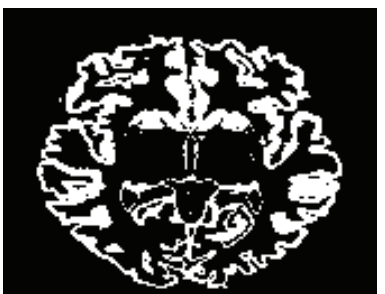

(k) $\lambda_{2}=2.0$

Figure 11: Demonstration of the impact of parameter $\lambda_{2}$ on the size of GM segmented by BCEFCM when $\lambda_{1}$ and $\lambda_{3}$ are both set to be 2.0

90-th image slice of BrainWeb which is corrupted by slight noise (3\%) and severe intensity inhomogeneity (60\% non-linear bias) with $\rho$ taking odd values in $[1,11]$ and give the results in Fig. 12 . We can see that the estimated bias fields are smooth enough and the bias corrected images are good enough if the parameter $\rho$ of BCEFCM takes values that are not less than 7 .

\section{Conclusion}

We have presented a fully automated method for segmentation of brain tissues in longitudinal MR images. The proposed method is able to segment brain tissues consistently from an arbitrary number of 


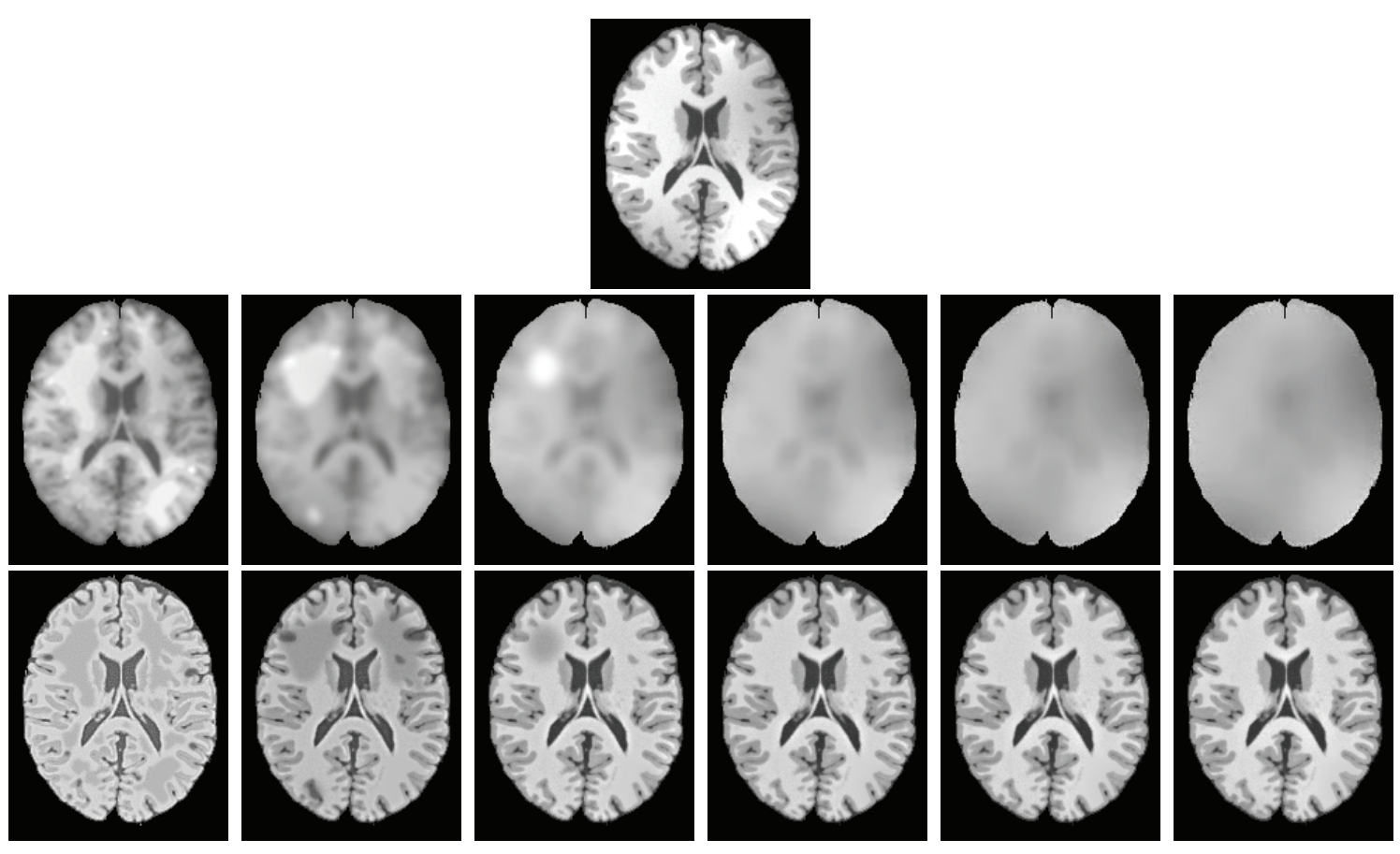

Figure 12: Demonstration of the impact of parameter $\rho$ on the smoothness of the estimated field and on the quality of the corrected image. The original image is given in the 1st row. The estimated bias fields and corrected images obtained from BCEFCM with parameter $\rho=1,3,5,7,9,11$ are given in the 2 nd and 3rd rows from left to right, respectively.

brain MR image series with the ability of dealing with the intensity inhomogeneities and overcoming the influence of noise. Quantitative evaluation and comparison with the state-of-the-art methods on BrainWeb images demonstrate the advantages of the proposed method, especially in terms of segmentation accuracy and consistency.

\section{Acknowledgements}

This work was supported by the Fundamental Research Funds for the Central Universities of China under grant N140403006, N140402003, and N140407001, the National Science Foundation for Distinguished Young Scholars of China under Grant No. 71325002, No. 61225012, the National Science Foundation of China under grant No. 61172002 and No.71071028, the Postdoctoral Scientific Research Funds of Northeastern University under Grant No.20150310, the National Key Technology Research and Development Program of the Ministry of Science and Technology of China under grant 2014BAI17B01, and the Fundamental Research Funds for State Key Laboratory of Synthetical Automation for Process Industries under Grant No.2013ZCX11. The authors would like to thank the editor for holding and considering to publish our paper and would also like to greatly appreciate the anonymous reviewers for their valuable comments to improve the quality of this paper. 


\section{References}

[1] M. G. Dwyer, N. Bergsland, R. Zivadinov, Improved longitudinal gray and white matter atrophy assessment via application of a 4-dimensional hidden Markov random field model, Neuroimage 90 (2014) 207-217.

[2] V. M. Anderson, J. M. Schott, J. W. Bartlett, K. K. Leung, D. H. Miller, N. C. Fox, Gray matter atrophy rate as a marker of disease progression in AD, Neurobiology of aging 33 (7) (2012) 1194-1202.

[3] F. Shi, Y. Fan, S. Tang, J. H. Gilmore, W. Lin, D. Shen, Neonatal brain image segmentation in longitudinal MRI studies, Neuroimage 49 (1) (2010) 391-400.

[4] C. Feng, C. Li, D. Zhao, C. Davatzikos, H. Litt, Segmentation of the left ventricle using distance regularized two-layer level set approach, in: Medical Image Computing and Computer-Assisted Intervention-MICCAI 2013, Springer, 2013, pp. $477-484$

[5] C. G. Schwarz, Longitudinal Tissue Segmentation Methods for the Aging Brain, University of California, Davis, 2012.

[6] R. Wolz, R. A. Heckemann, P. Aljabar, J. V. Hajnal, A. Hammers, J. Lötjönen, D. Rueckert, A. D. N. Initiative, et al., Measurement of hippocampal atrophy using 4D graph-cut segmentation: application to ADNI, NeuroImage 52 (1) (2010) $109-118$.

[7] S. F. Eskildsen, P. Coupé, V. Fonov, J. V. Manjón, K. K. Leung, N. Guizard, S. N. Wassef, L. R. Østergaard, D. L. Collins, A. D. N. Initiative, et al., BEaST: Brain extraction based on nonlocal segmentation technique, NeuroImage 59 (3) (2012) 2362-2373.

[8] C. Feng, D. Zhao, M. Huang, Image segmentation using CUDA accelerated non-local means denoising and bias correction embedded fuzzy c-means (BCEFCM), Signal Processing 122 (2015) 164-189, http://dx.doi.org/10.1016/j.sigpro. 2015.12 .007

[9] C. Davatzikos, F. Xu, Y. An, Y. Fan, S. M. Resnick, Longitudinal progression of Alzheimer's-like patterns of atrophy in normal older adults: the SPARE-AD index, Brain 132 (8) (2009) 2026-2035.

[10] Z. Yi, A. Criminisi, J. Shotton, A. Blake, Discriminative, semantic segmentation of brain tissue in MR images, in: Medical Image Computing and Computer-Assisted Intervention-MICCAI 2009, Springer, 2009, pp. 558-565.

[11] A. Mayer, H. Greenspan, An adaptive mean-shift framework for MRI brain segmentation, IEEE Transactions on Medical Imaging 28 (8) (2009) 1238-1250.

[12] Y. Zhang, M. Brady, S. Smith, Segmentation of brain MR images through a hidden Markov random field model and the expectation-maximization algorithm, IEEE Transactions on Medical Imaging 20 (1) (2001) 45-57.

[13] B. Scherrer, F. Forbes, C. Garbay, M. Dojat, Distributed local MRF models for tissue and structure brain segmentation, IEEE Transactions on Medical Imaging 28 (8) (2009) 1278-1295.

[14] Y. Wang, S. M. Resnick, C. Davatzikos, Analysis of spatio-temporal brain imaging patterns by hidden markov models and serial MRI images, Human brain mapping 35 (9) (2014) 4777-4794.

[15] M. L. Callisaya, R. Beare, T. G. Phan, L. Blizzard, A. G. Thrift, J. Chen, V. K. Srikanth, Brain structural change and gait decline: A longitudinal population-based study, Journal of the American Geriatrics Society 61 (7) (2013) $1074-1079$.

[16] H. Vrenken, M. Jenkinson, M. Horsfield, M. Battaglini, R. Van Schijndel, E. Rostrup, J. Geurts, E. Fisher, A. Zijdenbos, J. Ashburner, et al., Recommendations to improve imaging and analysis of brain lesion load and atrophy in longitudinal studies of multiple sclerosis, Journal of neurology 260 (10) (2013) 2458-2471.

[17] F. Shi, P.-T. Yap, J. H. Gilmore, W. Lin, D. Shen, Spatial-temporal constraint for segmentation of serial infant brain MR images, in: Medical Imaging and Augmented Reality, Springer, 2010, pp. 42-50.

[18] R. de Boer, H. A. Vrooman, M. A. Ikram, M. W. Vernooij, M. Breteler, A. van der Lugt, W. J. Niessen, Accuracy and reproducibility study of automatic MRI brain tissue segmentation methods, Neuroimage 51 (3) (2010) $1047-1056$.

[19] J. de Bresser, M. P. Portegies, A. Leemans, G. J. Biessels, L. J. Kappelle, M. A. Viergever, A comparison of MR based segmentation methods for measuring brain atrophy progression, Neuroimage 54 (2) (2011) 760-768. 
[20] J. Jovicich, M. Marizzoni, R. Sala-Llonch, B. Bosch, D. Bartrés-Faz, J. Arnold, J. Benninghoff, J. Wiltfang, L. Roccatagliata, F. Nobili, et al., Brain morphometry reproducibility in multi-center 3T MRI studies: A comparison of cross-sectional and longitudinal segmentations, NeuroImage 83 (2013) 472-484.

[21] L. Wang, F. Shi, G. Li, D. Shen, 4D segmentation of brain mr images with constrained cortical thickness variation, PloS one 8 (7) (2013) e64207.

[22] L. Wang, F. Shi, P.-T. Yap, W. Lin, J. H. Gilmore, D. Shen, Longitudinally guided level sets for consistent tissue segmentation of neonates, Human brain mapping 34 (4) (2013) 956-972.

[23] S. Roy, A. Carass, P.-L. Bazin, S. Resnick, J. L. Prince, Consistent segmentation using a Rician classifier, Medical Image Analysis 16 (2) (2012) 524-535.

[24] S. M. Smith, N. De Stefano, M. Jenkinson, P. M. Matthews, Normalized accurate measurement of longitudinal brain change, Journal of computer assisted tomography 25 (3) (2001) 466-475.

[25] S. M. Smith, Y. Zhang, M. Jenkinson, J. Chen, P. Matthews, A. Federico, N. De Stefano, Accurate, robust, and automated longitudinal and cross-sectional brain change analysis, Neuroimage 17 (1) (2002) 479-489.

[26] S. M. Smith, M. Jenkinson, M. W. Woolrich, C. F. Beckmann, T. E. Behrens, H. Johansen-Berg, P. R. Bannister, M. De Luca, I. Drobnjak, D. E. Flitney, et al., Advances in functional and structural MR image analysis and implementation as FSL, Neuroimage 23 (2004) S208-S219.

[27] B. Fischl, Freesurfer, Neuroimage 62 (2) (2012) 774-781.

[28] A. R. Khan, L. Wang, M. F. Beg, FreeSurfer-initiated fully-automated subcortical brain segmentation in MRI using large deformation diffeomorphic metric mapping, Neuroimage 41 (3) (2008) 735-746.

[29] Z. Xue, D. Shen, C. Davatzikos, CLASSIC: consistent longitudinal alignment and segmentation for serial image computing, Neuroimage 30 (2) (2006) 388-399.

[30] D. W. Shattuck, G. Prasad, M. Mirza, K. L. Narr, A. W. Toga, Online resource for validation of brain segmentation methods, NeuroImage 45 (2) (2009) 431-439.

[31] R. Suganya, R. Shanthi, Fuzzy c-means algorithm-a review, International Journal of Scientific and Research Publications 2 (11) (2012) 1 .

[32] L. Liao, T. Lin, B. Li, MRI brain image segmentation and bias field correction based on fast spatially constrained kernel clustering approach, Pattern Recognition Letters 29 (10) (2008) 1580-1588.

[33] M. Ahmed, S. Yamany, A. Farag, T. Moriarty, A modified fuzzy c-means algorithms for bias field estimation and segmentation of MRI data, IEEE Transactions on Medical Imaging 21 (3) (2002) 193-199.

[34] S. Chen, D. Zhang, Robust image segmentation using FCM with spatial constraints based on new kernel-induced distance measure, IEEE Transactions on Systems, Man, and Cybernetics, Part B: Cybernetics 34 (4) (2004) $1907-1916$.

[35] M.-S. Yang, H.-S. Tsai, A gaussian kernel-based fuzzy c-means algorithm with a spatial bias correction, Pattern Recognition Letters 29 (12) (2008) 1713-1725.

[36] J. P. Pluim, J. A. Maintz, M. A. Viergever, Mutual-information-based registration of medical images: a survey, IEEE Transactions on Medical Imaging 22 (8) (2003) 986-1004.

[37] S. M. Smith, Fast robust automated brain extraction, Human brain mapping 17 (3) (2002) $143-155$.

[38] J. Gao, C. Li, C. Feng, M. Xie, Y. Yin, C. Davatzikos, Non-locally regularized segmentation of multiple sclerosis lesion from multi-channel MRI data, Magnetic resonance imaging 32 (8) (2014) 1058-1066.

[39] A. Buades, B. Coll, J.-M. Morel, Nonlocal image and movie denoising, International Journal of Computer Vision 76 (2) (2008) 123-139.

[40] J. V. Manjón, J. Carbonell-Caballero, J. J. Lull, G. García-Martí, L. Martí-Bonmatí, M. Robles, MRI denoising using non-local means, Medical Image Analysis 12 (4) (2008) 514-523.

[41] A. Buades, B. Coll, J.-M. Morel, A review of image denoising algorithms, with a new one, Multiscale Modeling \& Simulation 
4 (2) (2005) 490-530.

42] P. Coupé, P. Yger, C. Barillot, Fast non local means denoising for 3D MR images, in: Medical Image Computing and Computer-Assisted Intervention-MICCAI 2006, Springer, 2006, pp. 33-40.

477 [43] J. Yang, C. Feng, D. Zhao, A CUDA-based reverse gridding algorithm for MR reconstruction, Magnetic Resonance Imaging 31 (2) (2013) 313-323.

44] C. Feng, D. Zhao, CUDA accelerated uniform re-sampling for non-Cartesian MR reconstruction, Bio-Medical Materials and Engineering 26 (s1) (2015) 983-989.

[45] C. Feng, J. Yang, D. Zhao, J. Liu, CUDA accelerated method for motion correction in MR PROPELLER imaging, Magnetic Resonance Imaging 31 (8) (2013) 1390-1398. 